\title{
MASKS: MANAGING ANONYMITY WHILE SHARING KNOWLEDGE TO SERVERS
}

\author{
Robert Pinto, ${ }^{1}$ Lucila Ishitani, ${ }^{2}$ Virgílio Almeida, ${ }^{3}$ Wagner Meira Júnior,${ }^{3}$ \\ Fabiano A. Fonseca, ${ }^{3}$ and Fernando D. Castro ${ }^{3}$ \\ ${ }^{1}$ Universidade Federal de Minas Gerais \\ Belo Horizonte, Brazil \\ robert@dcc.ufmg.br \\ ${ }^{2}$ Pontifícia Universidade Católica de Minas Gerais \\ Universidade Federal de Minas Gerais \\ Belo Horizonte, Brazil \\ lucila@dcc.ufmg.br \\ ${ }^{3}$ Universidade Federal de Minas Gerais \\ Belo Horizonte, Brazil \\ $\{$ virgilio,meira,fabiano,fernando\}@dcc.ufmg.br
}

\begin{abstract}
This work presents an architecture that allows users to enhance their privacy control over the computational environment. Web privacy is a topic that is raising, nowadays, many discussions. Usually, people do not know how their privacy can be violated or what can be done to protect it. Among the generated conflicts, we would like to show up the one that happens between privacy and personalization: by one side, users appreciate the idea of receiving personalized services and do not approve the collection, tracing and analysis of their actions; by the other side, personalization services need this type of information in order to profile their users. The architecture presented in this article helps users to understand better how their privacy can be invaded and, at the same time, gives them a better control of their privacy, through anonymity, without preventing them from receiving personalized services.
\end{abstract}

Keywords: privacy, personalization, anonymity and framework

\section{INTRODUCTION}

With the growth and spread of Internet services, information systems have the means to collect and build detailed profiles of individuals that 
use those services. These profiles can be used by Web sites to offer personalized services to their users. These services include customized content and recommendations based on user past behavior and actual interests. Personalization brings benefits for both sides of a Web interaction: users and sites.

According to Kobsa [Kobsa, 2001], "customers need to feel they have a unique personal relationship with the business" and to confirm this idea, he presents the result of a survey that shows that sites that offered personalized services increased in $47 \%$ the number of new customers. This result also shows that personalization benefits sites. The increase in the number of customers tends to increase sales and revenues. However, the personalization needs of collecting and analyzing user data (this work is commonly called data mining) can characterize privacy invasion. Privacy in the information age could be characterized as the right of individuals to protect their ability to selectively reveal information about themselves [Rao and Rohatgi, 2000]. Many people that use the Web know that information is collected about them, but they are not certain of what and how much it is collected, neither for what it will be used. In light of this notion, it is evident that the current Web infrastructure represents a serious threat to the privacy of users of services in the Web.

So, there is a great conflict to solve: how can data be disclosed for Web sites, so that personalized services could be offered to users, without violating their privacy? In this work we address this problem of protecting users from companies and online services that collect data during Web browsing activities, without preventing them from receiving personalized services. This framework is called MASKS (Managing Anonymity while Sharing Knowledge to Servers) and is based on the idea of minimizing exposure of individual information by putting an "anonymity barrier" between the private data and Web services, filtering the information that flows across the barrier to the service. In order to evaluate this "filtered information" we made use of an important concept of information theory: the entropy. We compared the entropy of the set of requisitions generated without MASKS and with MASKS and discovered that, although MASKS changes the set of requisitions sent to a site server, this new set of requisition will still offer some value about user interests.

This paper is organized in the following way. Section 2 presents related work. Section 3 presents our proposed solution for the conflict between privacy and personalization. Main characteristics of MASKS' components are presented in sections 4 and 5. The experimental evaluation of MASKS and its results are presented in section 6 . And section 7 presents our conclusions and future work. 


\section{RELATED WORK}

There are some previous work that also proposes to solve the conflict between privacy and personalization.

Crowds [Reiter and Rubin, 1998], mix networks [Chaum, 1981] and Onion Routing [Reed et al., 1996]provide anonymity without requiring a unique third-party to forward requests. The basic idea is to hide the real originator of a request, with the cooperation of the members of a group. In Onion, the path of message through the members of a group is pre-determined whereas in Crowds, a path is configured during transmission of a request. As all anonymity tools, both mechanisms do not allow personalization, due to the complete absence of identifiable information available to the web sites. But, there is a special case of anonymity that creates conditions to solve this problem: the persistent anonymity [Goldberg et al., 1997]or pseudonymity.

Pseudonyms are persistent user identification that, logically, can not be connected to the user real identification, but allows the association of a set of requests to a unique user. Lucent Personalized Web Assistant (LPWA) ${ }^{1}$ is the most known tool based on the use of pseudonyms. LPWA users can use the same pseudonym each time they return to a site, but they will have different pseudonyms for different sites. It allows Web sites to offer any kind of service that relies on user identification, without linking users to their actual identities. But, just like in real life, if someone discovers the real identity of a user behind a pseudonym, all user's past actions will be automatically exposed.

Arlein et al. [Arlein et al., 2000]proposed an architecture of shared user profiles, so that service providers could customize contents based even on user activities on other sites. This architecture preserves privacy because users can specify which information could be shared and grouped to form a profile. But this proposal is static or, in other words, it does not automatically adapt to changes on user interests.

Another option people have is to hide themselves behind a mask or personae. A digital persona is a model of the public personality of a person. A person can have many masks. Each of them reflects a specific role or interest that a person can have [Clarke, 1994]. Persona [Dunn et al., 1997] is a mechanism that is based on this idea. Each Persona is a personal data collection that a client can distribute to sites.

It protects privacy, because users controls what they accept to disclose to sites. The disadvantage of the mechanism is that it requires that users previously specify which information they accept to disclose. Besides

\footnotetext{
$\overline{{ }^{1} \text { http://www.bell-labs.com/projects/lpwa }}$
} 


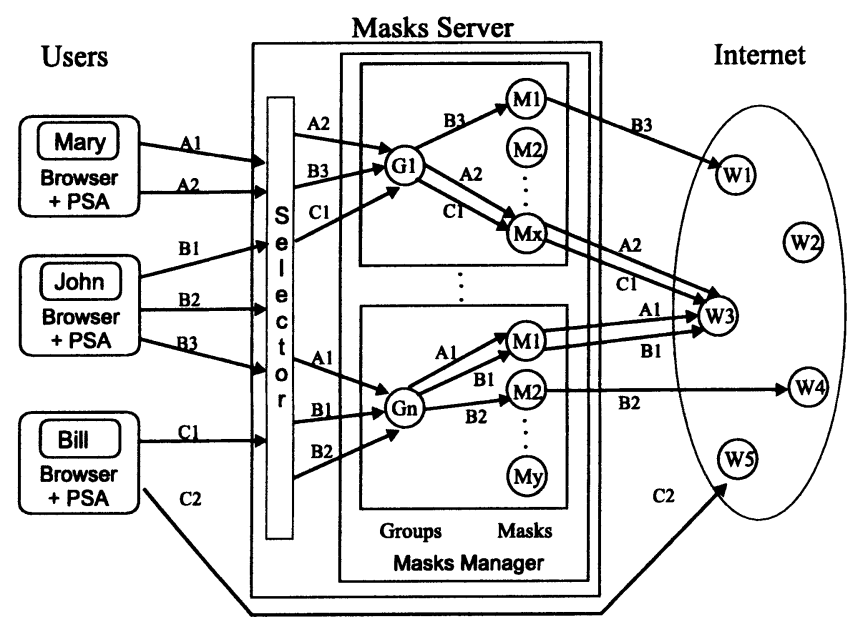

a) User requests

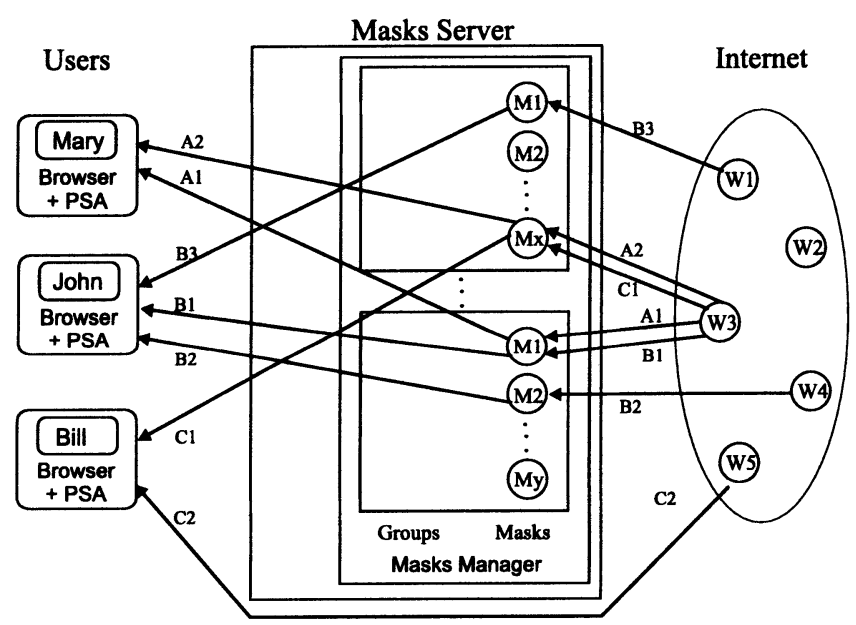

b) Site responses

Figure 1. Simplified architecture of MASKS

that, it does not adapt to dynamic aspects of user navigation. The profile it considers is restricted to static data, like name, birth date, address, and so on.

MASKS project proposes to solve the disadvantages of the architectures and technologies presented above. 


\section{MASKS}

This section describes MASKS (Managing Anonymity while Sharing Knowledge to Servers) [Ishitani et al., 2003](Figure 1), a Web-based framework that offers users a privacy-preserving mechanism, through anonymity. Unlike other anonymity tools, it allows some degree of personalization, for it discloses data that can be used by Web sites to provide personalized services, without profiling each individual user.

\subsection{Design characteristics}

MASKS's process is safe for users, because as it depends only on the last user request to function, it does not need to keep user data. The rationale behind this design decision is that the larger the amount of user information kept, the higher the probability that the server becomes a target of an attack.

Besides protecting user privacy, MASKS offers other benefits to users:

- Partial personalization compatibility - although being an anonymity mechanism, MASKS changes user request in a way that lets Web sites personalize services to users.

- Efficiency - the algorithms implemented are efficient in response time, because although the data structures used are complex, the applied mechanism is very simple. So, we expect that users will not perceive any latency when using MASKS;

- Dynamic - MASKS easily adapts to changes on user interests;

- Easiness of use - users do not need to provide any previous information to MASKS. No special protocol or proprietary technology is required, as MASKS employs standard HTTP and TCP protocols and work with usual identification mechanisms, such as cookies;

- Cover of a broader view of privacy protection - considering the six layers of privacy protection (awareness, control, privacy-enhancing tools, privacy policy, privacy and trust certification and privacy protection laws) [Ishitani et al., 2003], MASKS is the only architecture we know that implements the first three layers.

\subsection{MASKS Architecture}

The architecture protects user privacy through the use of masks. A mask is a temporary identification that a user may adopt while interacting with a Web site. This identification is associated with a user interest in a given topic or specific Web site. So, as users may present distinct 
interests while navigating in the Web, different masks can be associated with the same user even when they interact with the same site. For example, suppose that Web site W3, in Figure 1, is a portal that offers different classes of information such as travel and sports. Suppose also that Mary requests travel-related services (A1) and later requests sport information (A2). W3 would view the two requests from Mary (A1 and A2) as coming from two distinct users.

The assignment of masks to users is based on the concept of group. A group represents a topic of interest, and a user request is assigned to a group according to the semantics of the requested object. As an example, in Figure 1, requests A2 of Mary and C1 of Bill are assigned to the same group. Like with Crowds [Reiter and Rubin, 1998] and mix networks [Chaum, 1981], where web requests are not submitted by the individual user but by a user group (the Crowd) or by a mix network, user privacy is preserved. But differently from Crowds and Mix networks, MASKS provides data about the users' interests. These data can be used by Web servers to personalize the interaction.

Each group may have several masks associated with it, one for each site that offers the type of information associated with the group. For example, there are many sites that provide information about travel. The group associated with the subject travel will have one mask for each of the known sites related to travel. In Figure 1, this situation is represented by the requests $\mathrm{B} 1$ and $\mathrm{B} 2$ of John.

MASKS architecture has two major components: the Privacy and Security Agent (PSA) and the Masks Server. PSA is a program that each user runs in conjunction with the browser, keeping him/her informed about his/her potential privacy risks. PSA also allows users to turn off the masking process, if they want to interact directly with sites, without anonymity (request $\mathrm{C} 2$ in Figure 1).

The second component is the Masks Server, which is an intermediary that sits between users and Web sites, working as a proxy. It is responsible for managing masks and assigning them to the users.

\section{PRIVACY AND SECURITY AGENT}

Privacy and Security Agent (PSA) is a plugin, acting as an intermediary between users, Masks Server and Web sites. PSA receives user requisitions and resend them to Masks Server or to Web sites, according to user wishes of being masked or not. It is also a PSA function to receive sites' responses and to transmit them to users, with a privacy risk evaluation. 


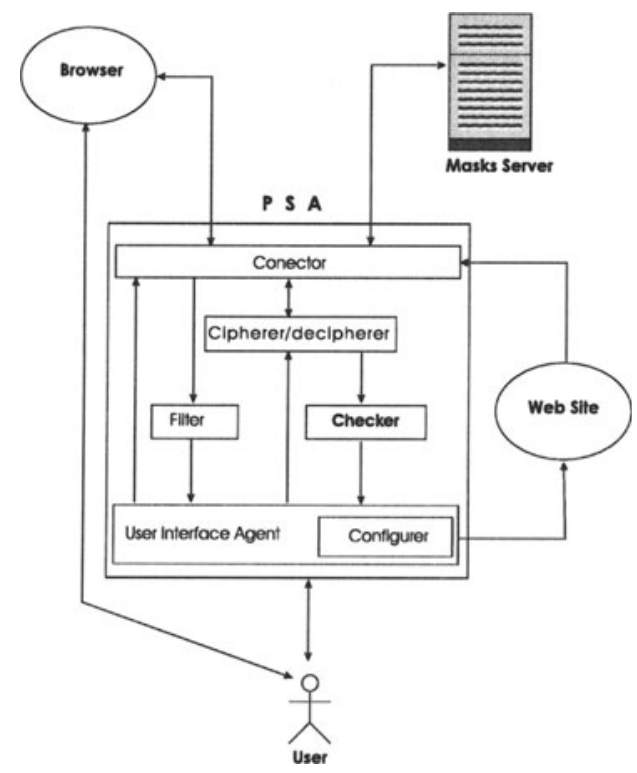

Figure 2. Simplified architecture of PSA

\subsection{PSA Architecture}

The main modules which compose PSA architecture are (Figure 2):

Conector: Module that acts as an intermediary between the user's browser and Masks Server. This module intercepts requests sent by the users to a Web site. It is also responsible to transmit responses of Masks Server to the user's browser, after their processing by the other modules of PSA.

Cipherer/decipherer: Module that ciphers and deciphers URLs sent to and received from a Masks Server. It aims to prevent thirdparties from having access to user private information, such as: accessed sites, searched topics, the IP address of a set of transmitted data.

Filter: Module that filters HTTP headers. Examples of information that can be filtered: browser identification and the page that the user was visiting when he/she sent the last request (referer).

Checker: Module that blocks known mechanisms of information collection, like Web Bugs.

User Interface Agent: This module keeps users informed about their privacy risks. It includes another module: the Configurer. 
Configurer: The function of this module is to allow users to choose the type of interface they want to use with PSA (pop-up windows or icons) and if they want to interact with Web sites behind masks or not.

The PSA processing of a user request is as following: first of all, the request is filtered. If any problem is detected, the user is informed about that and he/she can opt to continue his/her navigation masked or not. Before sending a filtered request to a Masks Server, PSA ciphers it.

The inverse process, or the PSA processing of a response sent by a Masks Server, starts with deciphering of the response. After that, the deciphered response is checked and "cleaned" by the Checker module. If any problem is detected, the user is informed. The response will only be transmitted to the browser, after satisfying PSA and user requirements.

\subsection{Implementation}

The first version of PSA prototype was implemented for the Mozilla browser. This decision was based on the fact that this browser makes easier the implementation of plugins and user interface, through the use of a language known as XUL (XML-based User Interface Language).

The following components were implemented through a $X P C O M$ architecture: the conector, the filter and, partially, the checker and configurer. Those components actions are activated by a user interface available on the browser interface.

\section{MASKS SERVER}

Masks Server is the main component of the MASKS architecture, as it is the responsible for the masks managing. It is the intermediary between PSA and Web sites, acting as a proxy.

Selector is the Masks Server component, responsible for selecting the interest group of each user requisition. Since the masks are divided into groups, one key issue for the effectiveness of the server is the definition of how objects are assigned to groups. One strategy we evaluated is the use of a semantic tree, more specifically the tree defined by the Open Directory Project ${ }^{2}$. This tree organizes Web sites based on a humanbased classification procedure. It is costless and completely available, representing a start point to define the groups and their relationships to each other. Each node of the tree represents a semantic category, or

${ }^{2}$ http://dmoz.org 


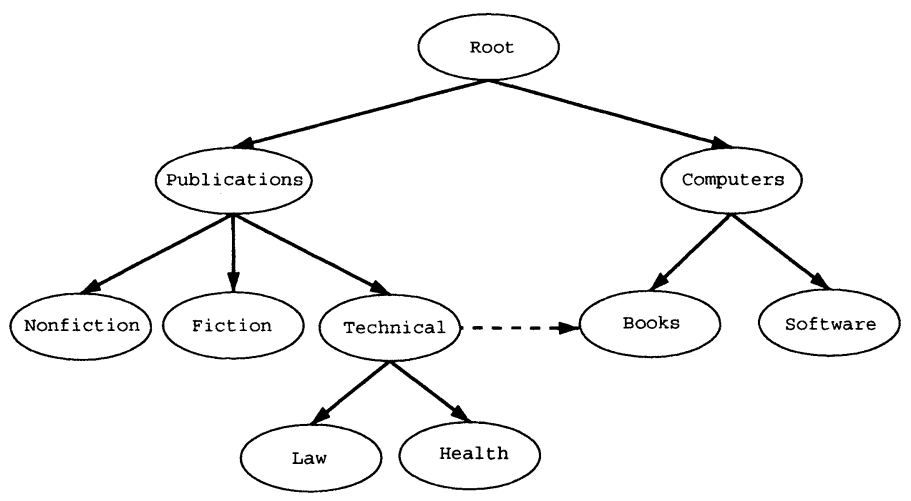

Figure 3. Example of a semantic tree

a group in our approach. Figure 3 illustrates an example of a semantic tree.

\subsection{Implementation}

For the implementation of a prototype of Masks Server we chose $S_{\text {quid }}{ }^{3}$. Squid is the result of the work of many people from the Internet Community, coordinated by Duane Wessels, of the National Laboratory for Applied Network Research. This architecture has the following advantages: its code is free and open, it works as an HTPP proxy, its scalability level is satisfactory. Besides these, Squid easily allows the capture of HTTP headers, which facilitate the processing of cookies sent by Web servers.

5.1.1 Cookie Processing. Cookie processing is shown in Figure 4. Figure 4-(a) represents a request sequence directly sent to a Web server, by the users John and Mary. Figure 4-(b) represents the same request sequence, with a Masks Server as an intermediary. In both situations, the Web server returns, with the response, a cookie that can be used for personalization services.

As can be seen, without using a Masks Server, users are directly receiving cookies. The Web Server asks for these cookies in the following requests. These cookies can be changed or not by the Web server. For example, in Figure 4-(a) the user John sends a new request along with the cookie ABC. The response he receives contains the new cookie ABD.

${ }^{3}$ http://www.squid-cache.org 


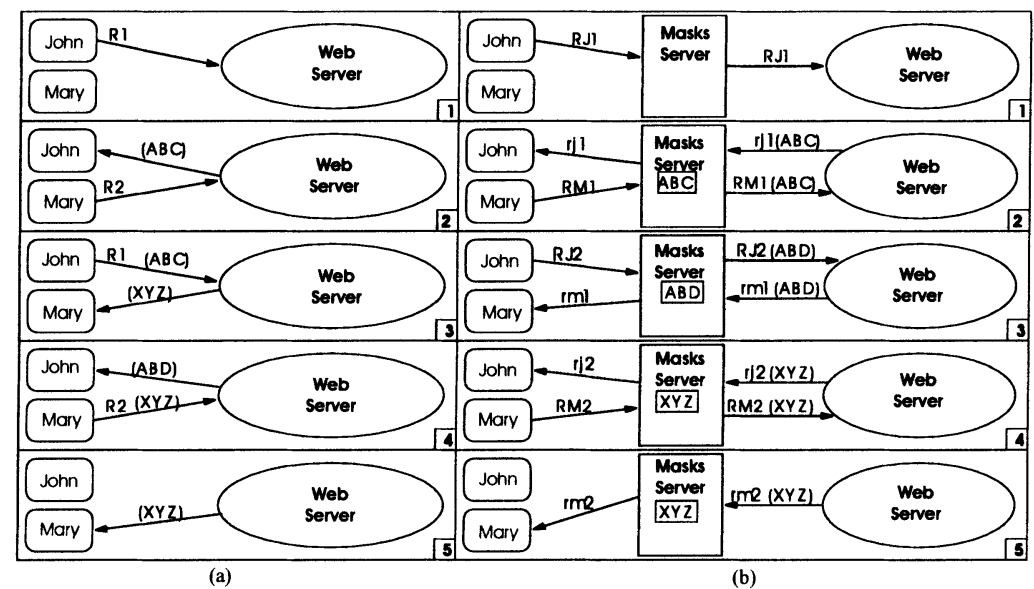

Figure 4. Cookie processing without/with a MASKS Server

By the other side, the Web server does not change the cookie XYZ associated to Mary's request.

When a Web user is using a Masks Server, this situation becomes different. The Web server will continue to do the same operations with the cookies, but the users will not receive the cookies. For example, suppose that in Figure 4-(b), John and Mary are interested on the same subject. According to the MASKS architecture, they both will be associated to the same interest group/mask. So, Masks Server resends user requests as if they belonged to a unique user. As can be seen in Figure 4-(b), the response to John's request $\mathbf{R J 1}$, will be $\mathbf{r j 1}$ along with the cookie ABC. When Mary sends her first request RM1, as it belongs to the same group of John's request, it will be resent to the Web Server, along with the cookie ABC. The response will be rm1, along with the cookie ABD. When John sends his second request RJ2, the Masks Server will resend it with the cookie ABD.

\section{EXPERIMENTAL EVALUATION}

In this section we present the methodology used to evaluate the quality of the data disclosed by MASKS to the Web sites. We also present the results of our experiments.

\subsection{Methodology}

It is hard to evaluate at what extent the data disclosed to Web sites will affect the personalization strategies adopted by the site, since there is a great number of different techniques that may be used. But it is feasible 
to estimate the worth of the disclosed information, which will always be the same regardless of the personalization strategy used by each Web site. According to the Information Theory the measure of the amount of information one variable contains is given by the entropy [Shannon, 1948].

In our case, as we want to discover if a set of masked requests will disclose valuable information to Web sites, the entropy will be related to the amount of information of each set. The smaller the occurrence probability of a request set, the bigger the entropy. And the bigger the occurrence probability of a request set, the smaller the entropy.

For calculating the entropies, we represented user navigation by a Markov model in which each state will represent a page and the links will represent page transitions, and their number of occurrences. Figure 5 illustrates the model adopted in our experiment and the entropy calculation.

In Figure 5-a), we present the basic subjects of seven pages of a fictitious site W. For each subject, MASKS will associate a different mask. Each page will have a occurrence probability, computed in the following way: $\quad p($ page $)=$ number of page requests / total number of requests to all pages of the site. In Figure 5-b), we can see that the total number of requests is 17 and that the number of requests to $\mathrm{P} 1$ is 3 . Thus, $p(P 1)=3 / 17 \approx 0,176$.

In Figure 5-b), we present a request sequence that arrives to the $\mathrm{W}$ site server, in two distinct days, including the user that sent the requests. In Figure 5-c), we present the same sequence, with masked users. In Figures 5-d) and 5-e), we present tables that detail the requests that compose sessions. In order to calculate the entropies, we build the models shown in Figures 5-f) and 5-g). These models were created based on the data of the Figures 5-d) and 5-e). Each node of the model represents one page and contains the number of times the page was accessed. Each link represents a page transition and has, associated to it, the number of times the transition has occurred. As the masking process changes the sessions, the models will have different entropies values.

The entropy calculation was based on the theory proposed in [Levene and Loizou, 1999]. The entropy value $H(M)$ of the model $M$ can be calculated by:

$$
H(M) \approx-\sum_{i=1}^{n} \sum_{j=1}^{n} \frac{m_{i, j}}{n} \log \frac{m_{i, j}}{m_{i}},
$$

where $n$ is the total number of pages that compose the model, $m_{i, j}$ is the total page transition number from page $i$ to page $j$ and $m_{i}$ is the total number of requsitions of page $i$. 


\begin{tabular}{|c|c|c|}
\hline Pages & Subject & Masks \\
\hline P1 & Sports & M1 \\
\hline P2 & Sports & M1 \\
\hline P3 & Finances & M2 \\
\hline P4 & Finances & M2 \\
\hline P5 & Finances & M2 \\
\hline P6 & Tourism & M3 \\
\hline P7 & Tourism & M3 \\
\hline
\end{tabular}

a) Pages and their masks

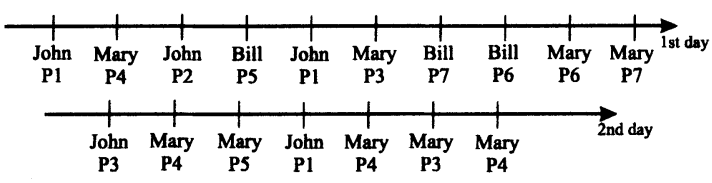

b) Sequence that original requests arrive at site server

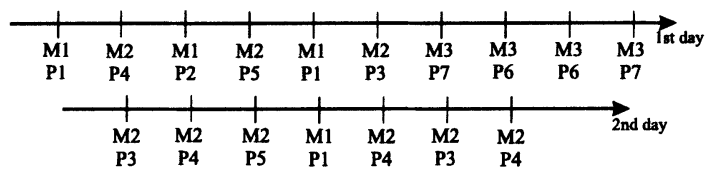

c) Sequence that masked requests arrive at site server

\begin{tabular}{|c|c|}
\hline User & Session \\
\hline John & P1, P2, P1 \\
\hline John & P3, P1 \\
\hline Mary & P4, P3, P6, P7 \\
\hline Mary & P4, P5, P4, P3, P4 \\
\hline Bill & P5, P7, P6 \\
\hline
\end{tabular}

d) Original sessions

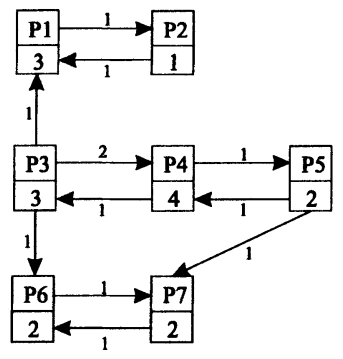

f) Navigation model for original requests (Model entropy $=1,37$ )

\begin{tabular}{|c|c|}
\hline User & Session \\
\hline M1 & P1, P2, P1 \\
\hline M1 & P1 \\
\hline M2 & P4, P5, P3 \\
\hline M2 & P3, P4, P5, P4, P3, P4 \\
\hline M3 & P7, P6, P6, P7 \\
\hline
\end{tabular}

e) Masked requests sessions
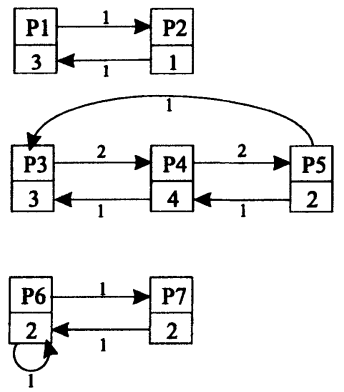

g) Navigation model for masked requests (Model entropy $=1,17$ )

Figure 5. Original and masked models and sessions

\subsection{Results}

The simulation was done using real logs of a virtual bookstore, collected during seven days. We have chosen the logs of a unique Web site and not a generalized log file, such as the one stored by a proxy, because we had to evaluate the value of the data disclosed for personalization from the point of view of a Web site. In other words, for offering per- 


\begin{tabular}{ccccc}
\hline Model & Entropy & IDs & Sessions & Avg. length \\
\hline Original & 8.01 & 227,047 & 242,990 & 1.52 \\
Masked (complete tree) & 4.72 & 12,105 & 166,345 & 2.22 \\
Masked (five-level tree) & 5.31 & 9,996 & 157,436 & 2.35 \\
Masked (four-level tree) & 5.97 & 8,780 & 147,459 & 2.50 \\
Masked (three-level tree) & 7.20 & 7,912 & 138,426 & 2.67 \\
Masked (two-level tree) & 7.79 & 7,580 & 131,858 & 2.80 \\
Masked (one-level tree) & 8.47 & 7,517 & 127,325 & 2,90 \\
Typically anonymized & - & 369,832 & 369,832 & 1 \\
\hline
\end{tabular}

Table 1. Results Comparison

sonalized services, Web sites must have access to user data (requests, profiles and navigation pattern). So, to discover if the personalization process will be affected, we have to study the difference between the original sessions of a site and the new masked one.

We simulated the requests for the following cases:

1 original user sessions;

2 masked sessions, considering different levels of the semantic tree sessions composed by requests whose client id field was replaced by the group mask. For the group assignment, we considered from one to five levels of the semantic tree and the complete semantic tree;

3 sessions composed by only one user request - this simulation was done in order to evaluate the result of other anonymizing mechanisms: each request is considered as being sent by a different user and because of that, we have sessions of only one request.

For each case we generated a distinct model. For each generated model, Table 1 presents some information about it: column Entropy shows the entropy of the model; column IDs, the number of different user identifications; column Sessions, the total number of sessions generated; Avg. length, the average session length. As expected, the entropies of the masked models are different from the entropy of the original model, but even so, the masked models offer more informational value than the typical anonymization process. It is not possible to calculate the entropy of the model generated by a sequence of typically anonymized requisitions. That is because the trails are composed of only one requisition and, as so, there are no transitions available to compute the entropy of user navigation. 
We can observe from Table 1, that the number of masked sessions and user IDs are smaller than those from the original model, because according to the masking process, the original requests are semantically grouped. This is a good result, because the more users mixed in a group, the more protected they are, as it will become more difficult to define the real profile of a specific user.

Table 1 also show us that there is no great difference between the masked models using some or all the levels of the semantic tree. It is clear that a greater number of levels contains a greater number of semantic groups. And, if there are more semantic groups, more different masks will be generated. This leads to a greater number of sessions, and a reduction on the average length of the sessions. But, as the semantic tree is very wide, even making use of only the first two of its levels, there will be a great variety of semantic groups. This result deserves attention, because it shows that there is no need to consider all the semantic tree to group user requests. Thus, the Masks Server can save some memory to store the groups and as it will have less semantic groups to analyze, the response time to resend a user request will be also smaller.

\section{CONCLUSIONS AND FUTURE WORK}

In this work, we show that, using MASKS, it is possible to disclose data that can be used to offer personalized services adapted to user interest without privacy invasion. The methodology used to prove it was based on the modeling of user navigation through a Markov chain model and the computation of the model entropy.

The results obtained emphasizes the usefulness of MASKS to the Web user activities of browsing and searching for specific information, which are the main points of privacy invasion [CyberDialogue, 1999] and the activities that spend most of the user time [Menascé et al., 1999].

As future work, it will be interesting to improve the masks association code and to evaluate other site classification strategies. There is also the need to develop a complete prototype of PSA.

Another future project is to implement a tree of Masks Servers that act cooperatively, exchanging information about semantic groups already known. This strategy will also provide the advantage of avoiding a single point of trust, as users will have more options of servers to provide them anonymity and their data can be distributed among different Masks Servers. 


\section{References}

[Arlein et al., 2000] Arlein, R. M., Jai, B., Jakobsson, M., Monrose, F., and Reiter, M. K. (2000). Privacy-preserving global customization. Proc. of Conference on Electronic Commerce (EC'00), pages 176-184.

[Chaum, 1981] Chaum, D. (1981). Untraceable electronic mail, return addresses, and digital pseudonyms. Communications of the ACM, 24(2):84-88.

[Clarke, 1994] Clarke, R. (1994). The digital persona and its application to data surveillance. The Information Society, 10(2).

[CyberDialogue, 1999] CyberDialogue (1999). American internet user survey: Privacy x personalization - part I. http://www.cybersitter.com.

[Dunn et al., 1997] Dunn, M., Gwertzman, J., Layman, A., and Partovi, H. (1997). Privacy and profiling on the web. Technical note, World Wide Web Consortium. http://www.w3.org/TR/NOTE-Web-privacy.html.

[Goldberg et al., 1997] Goldberg, I., Wagner, D., and Brewer, E. (1997). Privacyenhancing technologies for the internet. Proc. of IEEE Spring COMPCON. http://citeseer.nj.nec.com/54687.html.

[Ishitani et al., 2003] Ishitani, L., Almeida, V., and Meira Jr., W. (2003). Masks: Bringing anonymity and personalization together. IEEE Security $\&$ Privacy Magazine, 1(3):18-23.

[Kobsa, 2001] Kobsa, A. (2001). Tailoring privacy to users' needs. Proc. of 8 th International Conference on User Modeling. http://www.ics.uci.dcu/ kobsa/papers/2001-UM01-kobsa.pdf.

[Levene and Loizou, 1999] Levene, M. and Loizou, G. (1999). Computing the entropy of user navigation in the web. Research Note RN/99/42, Department of Computer Science, University College London.

[Menascé et al., 1999] Menascé, D. A., Almeida, V., Fonseca, R. C., and Mendes, M. (1999). A methodology for workload characterization for e-commerce servers. In 1st ACM Conference in Electronic Commerce (EC-99), pages 119-128.

[Rao and Rohatgi, 2000] Rao, J. R. and Rohatgi, P. (2000). Can pseudonymity really guarantee privacy? 9th USENIX Security Symposium.

[Reed et al., 1996] Reed, M. G., Syverson, P. F., and Goldschlag, D. M. (1996). Proxies for anonymous routing. In 12th Annual Computer Security Applications Conference, San Diego.

[Reiter and Rubin, 1998] Reiter, M. K. and Rubin, A. D. (1998). Crowds: Anonymity for web transactions. ACM Trans. on Information and System Security, 1(1):66-92.

[Shannon, 1948] Shannon, C. E. (1948). A mathematical theory of communication. The Bell System Technical Journal, 27:379-423, 623-656. 